$$
\begin{aligned}
& \left.\left(\frac{1}{e_{1}}-\frac{1}{\varepsilon_{2}}\right) \operatorname{tg} \alpha \frac{\partial u^{2 N}}{\partial z}\right|_{x=2 \operatorname{tg} \alpha} F(z \operatorname{tg} \alpha), \\
& F=\Psi_{m}, \Phi_{p}, \quad m=0,1, \ldots, N, \quad p=1,2, \ldots, N-1 .
\end{aligned}
$$

All the remaining matrices of the system can be evaluated with an accuracy of $O(\delta)$, by putting $\delta=0$.

In view of the stability of the boundary value problem $/ 4 /$, for small values of $\delta$ we can use the solution of problem (16) and (17) when $\delta=0$.

\title{
REFERENCES
}

1. SVESHNIKOV A.G., The incomplete Galerkin method, Dokl. Akad. Nauk SSSR, 236, 5, 1076-1079, 1977.

2. SVESHNIKOV A.G., IL'INSKII A.S, and KOTIK I.P., Propagation of waves in ixregular waveguides with sides of complex form, in: Computing Methods and Programming, Izd. MGU, III, 329-363, 1965.

3. ZAV'YALOV YU.S., KVASOV B.I. and MIROSHNICHENKO V.L., Methods of Spline-Functions, Nauka, Noscow, 1980 .

4. IL'INSKII A.S. and SITSHAYEVA Z.Z., The stability of the incomplete Galerkin method for calculating the propgation of normal modes in a plane waveguide, Vestnik MGU, Ser. 15, Computational Mathematics and Cybernetics, 2, 14-20, 1983.

Translated by R.C.G.

\section{A FINITE-DIFFERENCE METHOD FOR SOLVING THE EQUATIONS OF GAS DYNAMICS USING ADAPTIVE NETS WHICH ARE DYNAMICALLY ASSOCIATED WITH THE SOLUTION*}

\author{
N.A. DAR'IN, V.I. MAZHUKIN and A.A. SAMARSKII
}

\begin{abstract}
A finite-difference method for solving non-stationary, spatially onedimensional problems of gas dynamics is proposed. The method is based on the use of grids which are adapted and obtained by a coordinate transformation which, in its turn, is determined by the required solution. An efficient numerical algorithm has been constructed on the basis of the proposed method which enables the accuracy of the calculations to be increased considerably with a simultaneous reduction in the total number of mesh points in the net by a factor of 2-5 compared with other methods.
\end{abstract}

\section{Introduction.}

The correct choice of the computational net in problems of mathematical physics is always the most important part of a numerical calculation. This choice is particularly important in problems of gas dynamics, the solutions of which differ in a wide variety of features.

Two approaches, the Lagrangian and the Euler /1/, have been used from the outset in the development of methods for the numerical solution of the equations of gas dynamics.

In the Lagrangian methods $/ 2,3 /$, the cells of the computational net are moved together with the fluid and the velocity of motion of the mesh points is therefore determined by the velocity of the hydrodynamic flow. This method of adaptation has been found to be convenient in the case of relatively smooth flows in which there are no large deformations. Its application in problems with free surfaces and surfaces of separation is the most effective. When there are significant deformations of the flow, there is a strong distortion of the cells of the mesh which leads to a reduction in the accuracy of the calculations and a reduction in the step size for the integration. When the amplitudes of the perturbing forces are too large, this may also give rise to the so-called "reversal" of some of the cells which leads to a loss of physical meaning in the results of the calculations. There are a number of mechanisms for the regularization of computational nets of the Lagrangian type (see /4/ and $15 /$, for example) with the aid of which it is possible to extend the domain of these methods successfully. However, it is not possible to remove the drawbacks of the Lagrangian approach completely.

In numerical methods based on the Euler approach $/ 1,6 /$, the mesh points of the computational net are fixed and do not change during the calculations, which enables one to treat flows with strong deformations. However, in problems where the behaviour of the 
solution is not previously known, the arrangement of the mesh points may turn out to be less than optimal which makes it necessary to use nets with an exceeaingly fine mesh size. This drawback can be partially compensated for by a preliminary non-uniform distribution of the coordinate lines, if certain a priori information is available concerning the behaviour of the solution. However, the structure of the non-uniform meshes in problems with a complex geometry for the solution domain is not a simple problem. A number of methods have been proposed for constructing the simplest nets which solely take account of the shape of the boundaries of the domain and do not change during their motion. These are either based on a geometric approach $/ 7 /$ or make use of the concept of the automatic transformation of the coordinates $/ 8,9 /$.

Both approaches have been used to develop methods for constructing nets which take account of the motion of the boundaries of the computed region. Differentiation of the elliptic system of equations with respect to time is proposed for this purpose in methods using a transformation of the coordinates /10/. In methods based on the geometric approach /11/, moving nets, which are closely connected with the motion of the boundary or with one of the special features of the solution, are introduced. In particular, the separation of a bow shock wave has been achieved with their help in $/ 11$ and $/ 12 /$.

Attempts to combine the advantage of the two approaches led to the development of mixed Euler-Lagrange /13-17/ and quasi-Lagrangian /18-20/ methods.

Attempte to make use of all the advantages possessed by the moving net, Lagrangian, Euler and mixed Euler-Lagrangian approaches has led in the final analysis to the development and use of adaptive net methods. These nets are dynamically linked to the solution (see the reviews $(21-23 /)$.

At the present time, methods for constructing adaptive nets are under intensive development and it is therefore still quite impossible to state a preference for any of them. The choice of the characteristic of the solution which is used as the parameter which controls the motion of the mesh points of the net is the key problem in all of the methods for constructing adaptive nets which are dynamically linked to the solution. The accuracy of the solution also depends on how the difference scheme and the method of constructing the difference net are associated. Taking account of these requirements a number of empirical approaches to the construction of difference nets with a controlled distribution of the mesh points has been proposed by various authors.

In moving finite-element methods $/ 24,25 /$, the system of difference equations obtained using the Galerkin projection method is used for these purposes. At the same time, the opinion has been expressed in /25/ that moving finite-element methods have an indisputable advantage over finite-difference methods which use adaptive nets.

Methods for constructing adaptive nets in finite-difference methods $/ 26-34 /$ differ in an even greater variety of ways. Methods of constructing adaptive nets based on variational approaches $126,27 /$, an equidistant distribution of the dependent variables $/ 28 /$, by taking account of the exror in the approximation $/ 29,30 /$ and the gradients of the numerical solution $131 /$, etc. (see $/ 32 /$, for example) have become widely used in finite-difference methods.

The fact that the processes involved in determining the solution and the motion of the mesh points in them are treated as if they were separate and realized autonomously should be added to the drawbacks of the approaches which have been enumerated above. Furthermore, the reciprocal link between the difference scheme and the method of adjusting the net which are used is specified empirically and, as a rule, extremely crudely. It appears to us that it is, in fact, for these reasons that oscillations of the net or the linked vibrations of the solution and the net, which have been repeatedly pointed out in different papers, arise frequently when solving problems.

It is also not completely rational to detemine the solutions in a domain where there are singularities by concentrating the mesh points in it. For example, the isolation of a shock wave using this approach $/ 32 /$ requires about $10^{2}$ mesh points.

A method for solving non-stationary one-dimensional problems of gas dynamics on an adaptive net, dynamically linked with the solution, which is free from the above-mentioned drawbacks, is proposed in the present paper. The approach which is described in an extension to gas-dynamic problems of the method for solving boundary value heat conduction problems 133/ and problems of the stefan type /34/ on adaptive nets.

\section{Differential formulation of the problem.}

we shall define the finding of the numerical solution of the equations of gas dynamics with the aid of an adaptive net which is dynamically linked with the solution as a method of solution in which the finding of the net functions and the coordinates of the mesh points are continuously linked. The main differences between the proposed approach and those which have been previously considered are as follows.

1. The adaptive net is constructed using a corresponding coordinate transformation. The actual form of the transformation is specified in terms of a certain function $Q$, the form of which is determined by the singularities of the solution of the problem under investigation.

2. A close reciprocal link between the required solution and the method of adjusting the computational net is introduced at the level of the differential model which, in the general case, is a non-linear system of partial differential equations. In this system some of the equations directly describe the phonomenon under investigation while the others describe the dynamics of the computational net which are determined, in their turn, by the evolution of the solution. In the limiting cases of these equations, nets either in Eulerian or Lagrangian variables are obtained from these equations.

we note that the actual form of the link between the equations of gas dynamics and the 
equation for the adjustment of the net is specified by analogy with the quasi-Lagrange approach $118 \%$

We will use the Eulerian system of coordinates $(x, t)$ as the initial system of coordinates in which the mathematical formulation of the phenomenon under investigation is carried out. Then, with the help of a transformation of the general form $x=f(q, t)$, we make the transition from physical space to the computational space. The system of non-stationary equations of gas dynamics in the unidimensional approximation in Fulerian variables has the form

$$
\begin{aligned}
& \frac{\partial \rho}{\partial t}+u \frac{\partial \rho}{\partial x}=-\rho \frac{\partial u}{\partial x}, \quad \frac{\partial u}{\partial t}+u \frac{\partial u}{\partial x}=-\frac{1}{\rho} \frac{\partial p}{\partial x}, \\
& \left.\frac{\partial}{\partial t}{ }^{\prime} \varepsilon+\frac{u^{2}}{2}\right)+u \frac{\partial}{\partial x}\left(\varepsilon+\frac{u^{2}}{2}\right)=-\frac{\partial}{\partial x}(p u) .
\end{aligned}
$$

In the new variables $g$ and $t$, the system of Eqs. (1.1) is written in the form

$$
\begin{aligned}
& \frac{\partial \psi}{\partial t}=-\frac{\partial Q}{\partial q}, \\
& \frac{\partial}{\partial t}(\psi u)=-\frac{\partial p}{\partial q}-\frac{\partial}{\partial q}(Q u), \\
& \frac{\partial}{\partial t}\left[\psi\left(e+\frac{u^{2}}{2}\right)\right]=-\frac{\partial}{\partial q}(p u)-\frac{\partial}{\partial q}\left[Q\left(\varepsilon+\frac{u^{2}}{2}\right)\right], \\
& \psi=\rho \partial x / \partial q, \\
& Q=\rho(u-\partial x / \partial t) .
\end{aligned}
$$

The actual form of the function $Q$ is determined by the rule selected for the coordinate transformation, that is, by the form of the function $f(q, t)$. We note that, in the Lagrangian, quasi-Lagrangian and mixed Eulex-Lagrange approaches, the function $f(q, t)$ is assumed to be known. In the construction of an adaptive net which is dynamically inked with the solution, it is necessary that the rule governing the coorainate transformation (the function $f(a, t)$ ) should be determined by the required solution. On account of this, it is necessary to specify the function $Q$ for the complete definition of system (1.2)-(1.5).

Expressions (1.2)-(1.3) are the equations of continuity for the motion and the energy, and (1.4) is the equation for the link between the variables $x$ and $q$. Eq. (1.5) can be written in the form

$$
\partial x / \partial t=u-Q / \rho,
$$

where $u$ is the velocity, $\rho$ is the density of the gas and $\rho$ is an arbitrary function of the solution. As is shown below, a considerable improvement in the solution of the afference equations can be achieved by selecting the function $Q$ in the form

$$
Q- \pm(\gamma p \rho)^{1 / 2}
$$

where $p$ is the pressure and $\gamma$ is the constant ratio of the specific heat capacities ( $\gamma>1$ ). The choice of the function $Q$ in the form (1.7) means that each mesh point of the difference net either moves along the $C_{+}$-characteristic or along the $C_{\text {-characteristic since, in the }}$ case of $(1.7), \mathrm{Eq} .(1.6)$ takes the form

where $c$ is the velocity of sound.

$$
\partial x / \partial t=u \pm c, \quad c=(\gamma p / \rho)^{1 / s}
$$

In a number of cases, such as, for example, when describing the motion of a contact discontinuity, it is convenient to select the function $Q$ in the form $Q m 0$. It is obvious that, in this case, the variable $q$ is identical to the Lagrangian mass variable $m=j p d x$. Moreover,

when the function $Q$ is chosen to be of the form $Q=p u$, we arrive at the Euler variables since, in this case, $\partial x / \partial t=0$.

Eqs. (1.4) and (1.5) can be used to determine the Euler variabic $x(q, t)$ and the change in the density $\rho$. However, from the computational aspect, it is more convenient to take another equation instead of (1.5). In order to obtain this equation, we differentiate the left- and right-hand sides of (1.5) with respect to the spatial variable $q$ and, by using (1.4), we arrive at the equation for determining the change in $\rho$ :

$$
\frac{\partial}{\partial t}\left(\frac{\psi}{\rho}\right)=\frac{\partial u}{\partial q}-\frac{\partial}{\partial q}\left(\frac{Q}{\rho}\right) .
$$

The system of gas-dynamic equations in the variables $q$ and $t$ finally has the form (1.2)-(1.4), (1.8).

\section{Selection of the function $\boldsymbol{Q}$.}

When constructing the function $Q$, it is necessary to take account of the singularities of the solution of the problem under consideration. For instance, the construction of an adaptive net for solving the stefan problem $/ 33 /$ with isolation of the position of the phase 
boundary, in the simplest case requires a uniform distribution of the mesh points in each phase subregion. As a rule, the sizes of the spatial steps in these subregions are quite different and depend on the velocity at which the phase front moves. A mesh point distribution which is almost uniform is attained at each instant of time in the two subregions (with an invariant overall amount over the whole range of integration with respect to $t$ ) by specifying the function $Q$ in the form of a diffusion flow $Q=-D \partial \psi / \partial q$, where $D$ is a coefficient, the value of which is selected during the calculation.

A class of problems exists, the solution of which within the domain or on its boundary is characterized by the occurrence of large gradients which, if the required accuracy of the numerical solution is to be attained, necessitate the use of a finer mesh than is used within the remainder of the domain. Automatic compression of the mesh can be achieved by a concentration of the mesh points in it with the aid of the function $Q$, specified in the form

$$
Q=-Q_{0} \frac{\partial}{\partial q}\left(\psi\left|\frac{\partial T}{\partial q}\right|\right)
$$

The removal of mesh points from a region where the solution only changes slightly can lead to a very irregular net in this region. In order to avoid this, it is necessary to use a smoothing mechanism and, for this purpose, the function $Q$, in problems with large gradients, has been specified in the form of the combination /33/:

$$
Q=-D \frac{\partial \psi}{\partial q}-Q_{0} \frac{\partial}{\partial q}\left(\psi\left|\frac{\partial T}{\partial q}\right|\right)
$$

In problems which describe complex processes, the form of the function $\Omega$ must, obviously, be determined by the predominant process. We note that, in evolutionary problems, the form of the equation used for readjusting the net must always be of the evolutionary type.

Gas-dynamic problems are distinguished by a wide variety of solutions and it is therefore difficult to indicate a universal form of the function $Q$ which would satisfy all problems. We will now consider some methods of specifying the function $Q$ in problems of gas dynamics using the solution of a number of actual problems as an example.

3. The difference scheme.

In the computed space $\Omega_{q_{t} t}$, we introduce a computational net $\omega$ with a constant step size $h$ with respect to the variable $q$ and a step size $\tau$ with respect to the variable $t:$

$$
\begin{aligned}
& \omega=\left\{\left(q_{i}, t^{j}\right),\left(q_{i+y_{3}}, t^{j}\right), q_{i+1}=q_{i}+h, q_{i+1 / 2}=q_{i}+0.5 h,\right. \\
& \left.t^{i+1}=t^{j}+\tau, \quad i=0,1, \ldots, N-1, \quad i=0,1 \ldots\right\} .
\end{aligned}
$$

On this net, the system of Eqs. (1.2)-(1.4), (1.8) is approximated by a family of difference schemes in which the functions $\psi, \rho, u, p$, and $\varepsilon$ are calculated at the half-integral points $\left(q_{i+1 / 4}, t^{i}\right)$ while the values of $x$ and $Q$ are calculated at the integral points $\left(q_{i}, t^{j}\right)$. The family of difference schemes has the form

$$
\begin{aligned}
& \frac{\psi_{i+1 / 2}^{j+1}-\psi_{i+1 / h}^{i}}{\tau}=-\frac{Q_{i+1}^{(o)}-Q_{i}^{(\alpha)}}{h} . \\
& \frac{(\psi / \rho)_{i+1 / 2}^{j+1}-(\psi / \rho)_{i+y_{2}}^{j}}{\tau}=\frac{u_{i+1}^{(0)}-u_{i}^{(0)}}{h}-\frac{(Q / \rho)_{i+1}^{(0)}-(Q / \rho)_{i}^{(0)}}{h}, \\
& \frac{\left[\psi\left(\varepsilon+u^{2} / 2\right)\right]_{i+1 / 2}^{j+1}-\left[\psi\left(\varepsilon+u^{2} / 2\right)\right]_{i+1 / 2}^{j}}{\tau}=-\frac{(p u)_{i+1}^{(0)}-(p u)_{i}^{(0)}}{h}- \\
& \frac{\left[Q\left(\varepsilon+u^{2} / 2\right)\right]_{i+1}^{(\sigma)}-\left[Q\left(\varepsilon+u^{2} / 2\right)\right]_{i}^{(\sigma)}}{h}, \\
& \left(x_{i+1}^{j+1}-x_{i}^{j+1}\right) / h=(\psi / \rho)_{i+1, b}^{j+1}
\end{aligned}
$$

The values of the functions $\psi, \rho, u, p$, and $\varepsilon$ at the integral mesh points were calculated using the interpolation formulae

$$
y_{i}=\frac{\psi_{i+y_{i}} y_{i-y_{2}}+\psi_{i-y_{1}} y_{i+y_{2}}}{\psi_{i-y_{2}}+\psi_{i+y_{2}}}
$$

When $\sigma=0$ and 1 , the difference scheme $(3.1)$ is of the order of approximation of $O\left(\tau+h^{2}\right)$ and, when $\sigma=0.5$, the order of approximations of $O\left(\tau^{2}+h^{2}\right)$. In the following calculations schemes, Eqs. (3.1) with $\sigma=0.5$ and 1 were employed. The implicit schemes were solved by the method of simple iterations.

Let us now consider the solution of several non-stationary, spatially one-dimensional problems of gas dynamics. The choice of these problems was dictated by an attempt to demonstrate the possibilities of the proposed method of solution as fully as possible.

4. The problem of the formation of a shock wave.

Let us consider the problem of the formation and propagation of a shock wave using the 
example of the motion of an accelerating piston. At the instant of time $t=0$, the piston occupies the position $x=0$ and then moves according to the law $x=a t^{2}, a>0$ in a tube filled with a quiescent polytropic gas. During the development of the compression wave, the gradients of the solution in this zone increase and, at a certain critical instant of time, $t=t_{c}$, become infinite. A so-called gradient catostrophy occurs, that is, a singularity occurs in the solution. The instant of the onset of the gradient catastrophy can be determined using the formula/35/

$$
t_{\mathrm{K}}=u_{\mathrm{c}} \%[(\gamma+1) a] \text {, }
$$

where $u_{c}^{0}$ is the velocity of sound in the quiescentgas and $a$ is the constant in the law of motion of the piston. For $\rho_{0}=1, p_{0}=1$, and $a=1$, the time $t_{\mathrm{C}}=0.484$, and $\gamma=5 / \mathrm{s}$.

Up to the instant of the onset of the gradient catastrophy, the flow is a simple compression wave with a weak discontinuity at the point where it joins with the constant flow. An attempt has been made to determine the instant when the discontinuity forms from the numerical solution of the system of finite difference Eqs. (3.1) with $0=0.5$ and 1 on a fixed Lagrangian net $(Q=0)$ with a total number of mesh points $N=60$. Spatial profiles of the velocity $u$ at different instants of time obtained using the implicit difference scheme $(\sigma=1)$ with first-order accuracy (Fig.1) and the scheme with second-order accuracy ( $0=0.5$ (Fig.2) are shown in Figs.l and 2. The numerical solution is shown by means of broken lines and the exact solution by the solid lines. The positions of the mesh points are indicated by the small circles. The results obtained suggest that it is practically impussible to determine the instant of time at which the shock wave is formed from numerical calculations on a fixed mesh. The solutions obtained using the first order scheme (Fig.l) do not reproduce the steep fronts on account of the large approximation viscosity of the scheme. The second-order scheme yields oscillations in regions where there are large gradients (Fig.2).

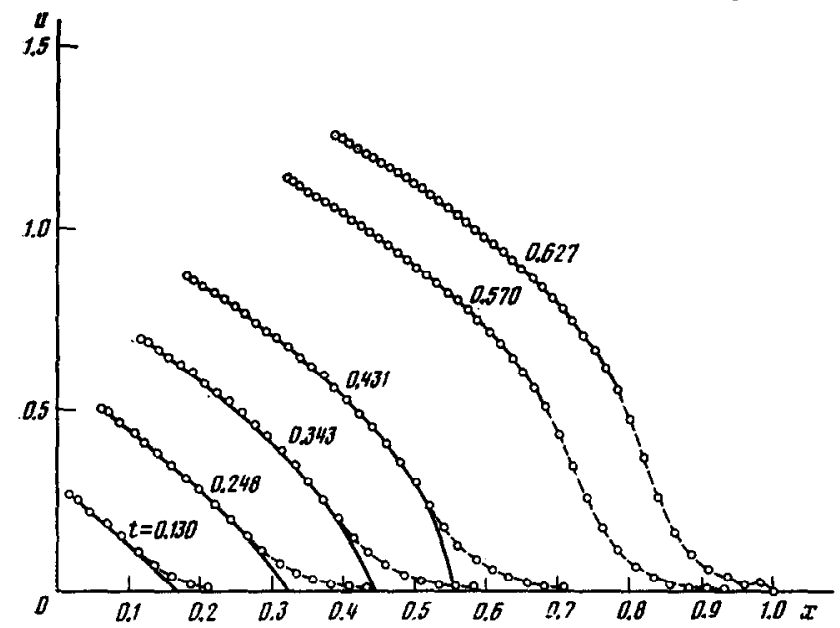

Fig.1

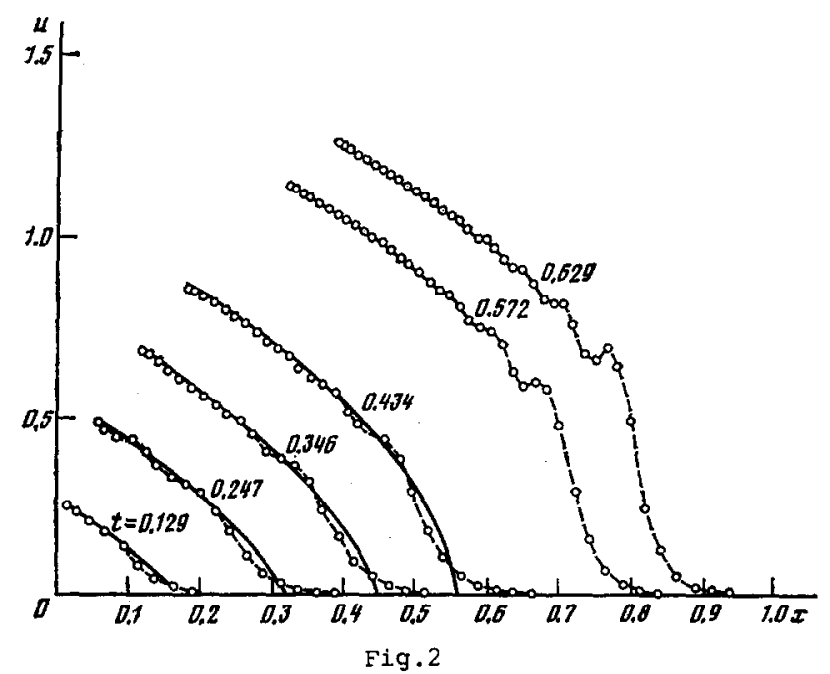

The qualitative effect of the approximation viscosity on the solutions which are obtained was investigated using the method of differential approximation $/ 36 /$. As an example, let us take the first differential approximation for the difference equation of motion from system (3.1) with $\sigma=0.5$ :

$$
\frac{\partial}{\partial t}(\psi u)+\frac{\partial p}{\partial q}+\frac{\partial}{\partial q}(Q u)=-\frac{1}{3 !}\left[\frac{\tau^{2}}{4} \frac{\partial^{3}(p+Q u)}{\partial t^{2} \partial q}+\frac{h^{2}}{8} \frac{\partial^{3}(p+Q u)}{\partial q^{3}}\right]
$$




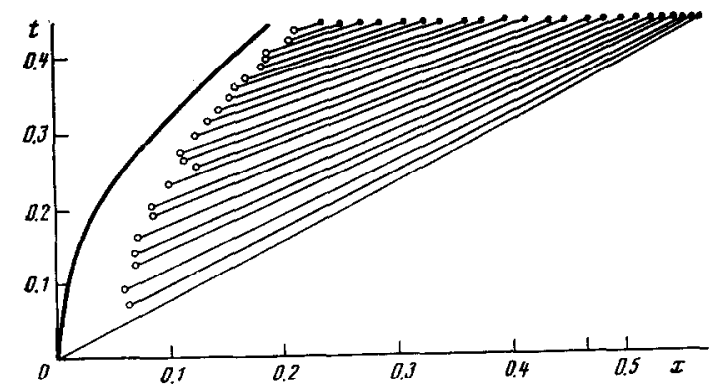

Fig. 3. $\quad(x-t)$-diagram

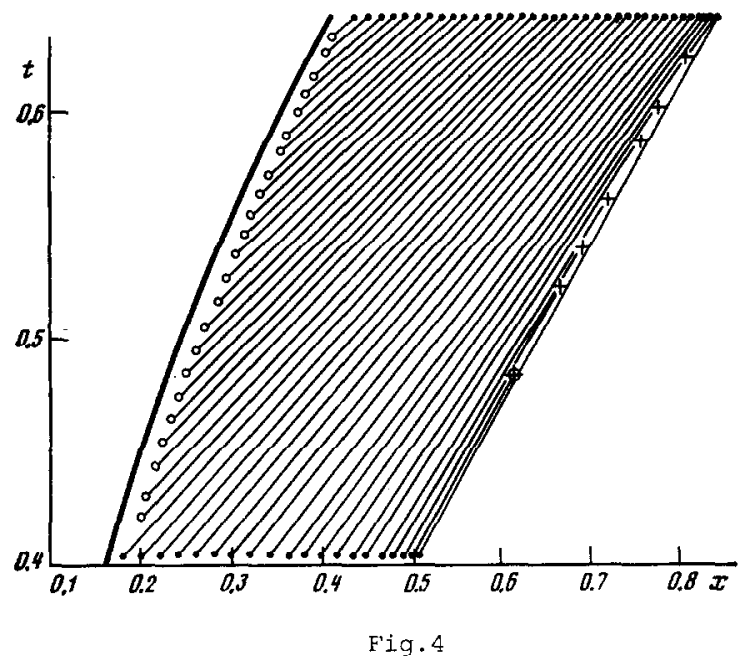

We note the following: the oscillatory nature of the behaviour of $u$ when $Q=0$ is obviously determined by the first term on the right-hand side of (4.1). Both of the terms on the righthand side are functions of the derivatives of the quantities $p+Q u$ with respect to the spatial coordinate $q$. It is therefore desirable, when solving a problem using an adaptive net for the mesh points, to specify a law of motion such that the derivatives of the quantity $p+Q u$, close to large gradients in the solution, are small. It is well-known $/ 37 /$ that, in the solutions of gas dynamic problems which are represented in the form of a simple wave, all the quantities are functions of just a single quantity which keeps a constant value on the characteristics. It is natural to select the function $Q$ such that the coordinates of the mesh points in the physical space should move along the corresponding family of characteristics. In the problem under consideration, this must be the family of $C_{+}$-characteristics, the equation of which is written in the form

$$
d x / d t=u+u_{\mathrm{c}}
$$

where $u_{\mathrm{c}}$ is the velocity of sound. A comparison of (4.2) with Eq. (1.5) showed that the Eulerian coordinate of a meshpoint $x$ moves along a $C_{+}$-characteristic, if

is chosen.

$$
Q=-\rho u_{\mathrm{c}}
$$

The instant when the compression wave becomes a shock wave can be determined by the following considerations. It is known from the method of characteristics /37/ that a gradient catastrophy in a simple compression wave implies the intersection of two characteristics of the corresponding family. In the problem under consideration, when the flow $Q$ is selected in the form (4.3), each mesh point of the net must move along a $C_{+}$-characteristic. The position and the instant of formation will be determined when the quantity $\psi$ vanishes in the cell between the two intersecting characteristics. The calculations confirmed the hypotheses which have been put forward. The results of these calculations are presented in Figs.3-7.

There are a number of important aspects associated with the formation of a shock wave and its subsequent propagation.

1. It is known /35/ that a gradient catastrophy occurs near the base of a compression wave when the characteristics interest. One of these characteristics emerges from the piston at the instant of time $t=0$, that is, from the origin of coordinates. When a net which is uniform with respect to $q$ and the initial values $\psi_{i}=1$ are used, such a characteristic does not occur in the calculations and this may lead to an appreciable error in determining the value of $t_{c}$. In our calculations, when a uniform mesh was used to approximate the characteristic emerging from the piston, the initial value of $\psi_{1}$ in the first cell was chosen to be 
much small than in the remaining cell: $\psi_{1}=10^{-4}, \psi_{i}=1, i=2,3, \ldots, N-1$. Satisfaction of the inequality $\phi_{i} \leqslant 0.1$ in a cextain cell $l$ was considered to be the condition for the formation of a shock wave. In our calculations, using the selected values of $u_{c} a$ and $\gamma$, it was satisfied at the instant of time $t=0.475$, which is close to the calculated $t_{\mathrm{C}}=0.484, \Delta t \approx 0.02 t_{\mathrm{C}}$.

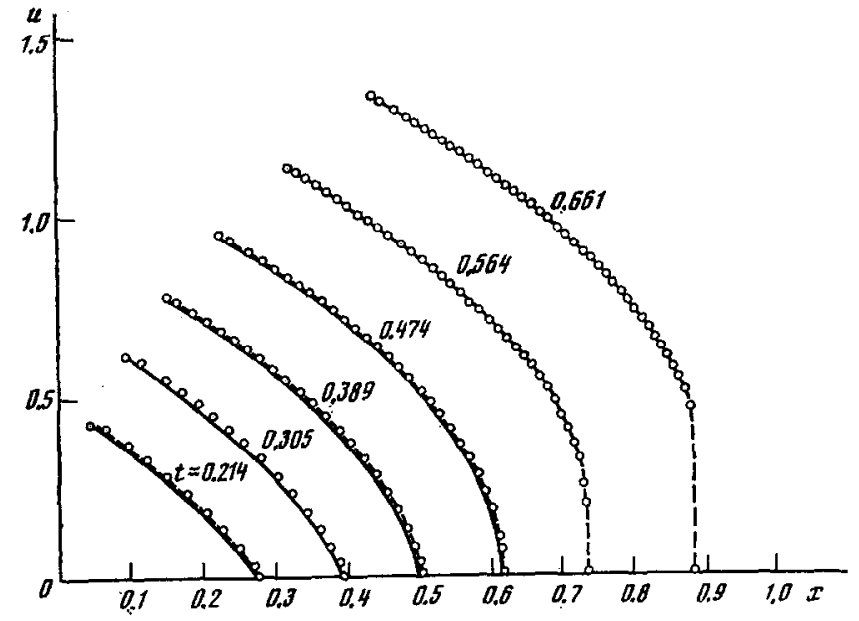

Fig. 5
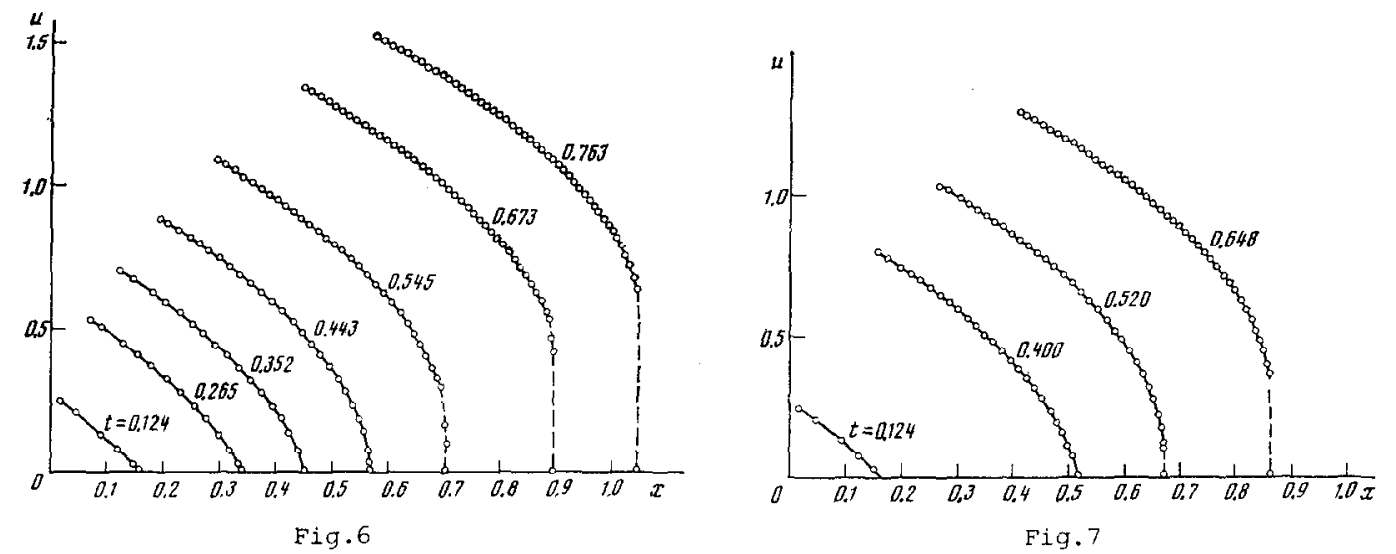

2. The boundary conditions

$$
Q\left(q_{0}, t\right)=Q\left(q_{R}, t\right)=0 .
$$

must be satisfied on the boundaries $q=q_{0}$ and $q=q_{R}$ of the computed region $q_{0} \leqslant q \leqslant q_{R}$.

Hence, when the mass overcurrent is chosen in the form of (4.3), the extreme left-hand cell will increase without limit while the extreme right-hand cell collapses to a point after a finite time. In order to prevent the above-mentioned phenomena from occurring, a mechanism for generating new cells close to the piston and anninilating the fine cells on the right-hand end of the region was incorporated in the numerical algorithm. The value of the quantity $\psi$ for the corresponding cell served as the criterion for annihilation or creation. In the rew cells, the values of $p$ and $u$ were determined by interpolation. The density $p$ was found from the requirement that the entropy should be conserved, and the function $\psi$ was determined from the law of conservation of mass. Trajectories of the motion of the mesh points of the net are shown in Figs. 3 and 4 . The mesh points used in the calculations are indicated by dots, the generated mesh points are indicated by small circles while those which are annihilated are indicated by small crosses. The instant when the shock wave is formed is indicated by a small circle with a cross inside it (Fig.4). The initial net consisted of the minimum number of celis, equal to two. The initial values of the function $\psi$ for these cells differed by several orders of magnitude. At the end of the calculations the number of mesh points had reached 40 (Fig.4).

3. After a strong discontinuity has been formed, the difficult question concerning the method of calculating the propagation of a non-stationary shock wave arises. There are two possibilities for picking out domains where there are singularities in the solution in calculations in adaptive nets. The space-time profile of a discontinuity can be reproduced with a sufficient degree of accuracy by concentrating a large number of mesh points in this region and, at the same time, not allowing any cells to collapse. However, this route is not entirely rational for two reasons. Firstiy, a large overall number of mesh points is required and, secondly, excessive densification of the net with respect to the spatial variable may 
bring about a substantial reduction in the integration step size with respect to time. sis result, the question arises of the efficiency of the method.

Another route is more promising when the singularities in the solution are isolated in an explicit form according to the type of solution of the stefar problem in $/ 34 /$ in which the discontinuity is located on a mesh point of the net. The boundary conditions in the form of conservation laws are written out on the discontinuity and one of these conservation lawe allows one to establish the mass flux through the discontinuity. The value of this flux is then used in order to construct the net which is adapted.

The isolation of a strong discontinuity means that it is located at a certain mesh point $i=l$, at which the values of $\rho_{i}, u_{l}, p_{i}$, and $\varepsilon_{l}$ are different to the left and right of thi. point. It is known that the Hugoniot relationships must be satisfiod wr this disconinuity. In the computational space they are written in a form which excludes the appearance of difference sources:

$$
\begin{aligned}
& u_{2}-Q / \rho_{2}=u_{1}-Q / \rho_{1}, \quad p_{2}+Q u_{2}=p_{1}+Q u_{1}, \\
& p_{2} u_{2}+Q\left(\varepsilon_{2}+u_{2}{ }^{2} / 2\right)=p_{1} u_{1}+Q\left(\varepsilon_{1}+u_{1}{ }^{2} / 2\right) .
\end{aligned}
$$

Here, the indices 1 and 2 denote values in front of and behind the shock wave respectively. The numerical calculations were found to be stable when the values frorn the region af constant flow $\rho_{1}, u_{1}$, and $\varepsilon_{1}$ and one of the values, $u_{2}$. for example, from the region beina the shock wave front were extrapolated onto the surface of the discontinuity. After the formation of a shock wave where a fixed mesh point of the computational net corresponds to tire position of its front, the cells to the right and the left of it were annihilated nsing the techruque which has been described above.

4. We will now consider the question of the link between the choice of 0 and the difference scheme. It is known/1-3/ that implicit schemes of the first order of accuracy on fixed nets greatly "blurs" weak discontinuities in a gas-dynamic flow. As a rule, secondorder schemes lead to the appearance of parasitic oscillations in the solution.

The use of adaptive nets, which are dynamically related to the solution, sulustantially changes these qualities of difference schemes. Calculations of first-order accuracy using the scheme (3.1) $(\sigma=1, F i g .5)$ and schemes of second-order accuracy $(\sigma=0.5$, Fig.6) showed that the blurring and oscillatory effects practically disappeared in both cases. The differcnco between the numerical solutions and the exact solutions did not exceed 1-2\%. The exact solution is shown by the solid lines, the numerical solution is shown by the broken lines and the positions and number of mesh points used are indicated by the small circles. $A$ comparison showed that the two schemes yield slightly different results. The instart at which the strong discontinuity arises was determined with an identical accuracy in the two cases. Both schemes clearly reproduce the discontinuity region when not more than 40 mes! points are used.

In order to ensure that all of this is also characteristic of other difference scherres, one of the widely used predictor-corrector type schemes, the Lax-wendroff scheme /1/ was employed for the purpose of comparing the calculations. The results obtained using this scheme are shown in Fig.7. They hardly differ from the results obtained using (3.1) either qualitatively or quantitatively.

It may therefore be conciuded that the use of the method based on the employment adaptive nets for solving problems in gas dynamics reduces the requirements regarding the difference schemes.

other qualities of the proposed method were investigated using the example of probiens which admit of selfmodelling solutions.

5. The problem of the propagation of a shock wave on a background with an exponentially increasing density.

As an example of this problem /38/ we shall carry out an additional experimental investigation of the accuracy of the numerical solution obtained on a net constructed with the help of the mechanisms for the generation and adaptation of the meshpoints described in sect. 4 .

At the initial instant of time $t=t_{0}$, the values of the gas-dynamic functions have the form $/ 16 /$

$$
\begin{aligned}
& u\left(x, t_{0}\right)=\left\{\begin{array}{cc}
0, & x \geqslant x_{0}, \\
\Delta\left(1-\xi_{0}\right) / t_{0}, & x \leqslant x_{0},
\end{array}\right. \\
& \varepsilon\left(x, t_{0}\right)=\left\{\begin{array}{cc}
0, & x \geqslant x_{0}, \\
0.5\left(\Delta / t_{0}\right)^{2}\left(1+2 \xi_{0}\right), & x \leqslant x_{0},
\end{array}\right. \\
& \rho\left(x, t_{n}\right)=\left\{\begin{array}{cc}
\rho_{0} \exp \left[\left(x-x_{0}\right) / \Delta\right], & x \geqslant x_{0}, \\
3 \rho_{0}\left(1+2 \xi_{0}\right)^{-3 / 2}, & x \leqslant x_{0} .
\end{array}\right.
\end{aligned}
$$

Here, $\xi_{0}=\left(x_{0}-x\right) / \Delta, x_{0}$ is the position of the discontinuity at the instant $t_{0}$, $\rho_{0}$ is the unperturbed background density when $x=x_{0}$ and $\Delta$ is the characteristic scale of the pulse width.

The selfmodeling solution when $t>t_{0}$ has the form

$$
u(x, t)=\left\{\begin{array}{cl}
0, & x \geqslant x_{F}(x), \\
\Lambda(1-\xi) / t, & x \leqslant x_{F}(t),
\end{array}\right.
$$




$$
\begin{gathered}
\varepsilon(x, t)=\left\{\begin{array}{cc}
0, & x \geqslant x_{F}(t), \\
0.5(\Delta / t)^{2}(1+2 \xi), & x \leqslant x_{F}(t),
\end{array}\right. \\
\rho(x, t)=\left\{\begin{array}{cc}
\rho_{0} \exp \left[\left(x-x_{0}\right) / \Delta\right], & x \geqslant x_{F}(t), \\
3 \rho\left(x_{F}\right)(1+2 \xi)^{-3 / 2}, & x \leqslant x_{F}(t) .
\end{array}\right.
\end{gathered}
$$

Here, $x_{F}(t)=x_{0}+1.5 \Delta \ln \left(t / t_{0}\right)$ is the position of the front at the instant $t$ and $t, \xi=\left[x_{F}(t)-x\right] / \Delta$. The numerical values of the parameters were as follows: $t_{0}=2, x_{0}=6, \rho_{0}=1, \Delta=4, \gamma=2$.
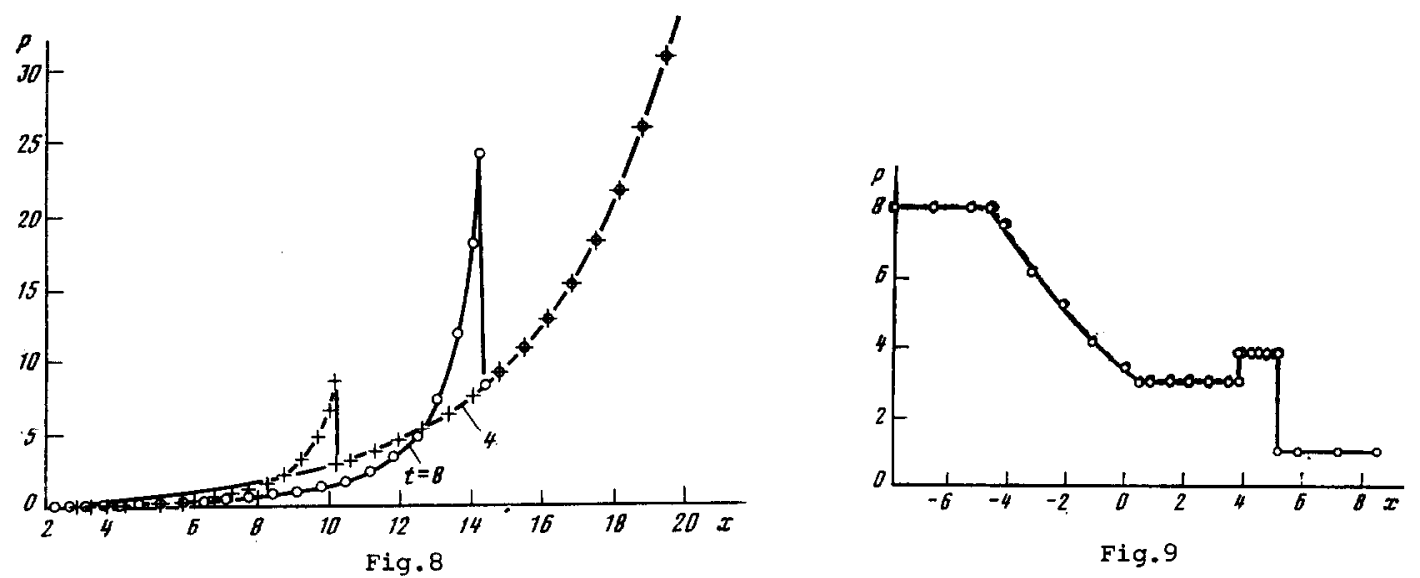

Results of calculations using the above-mentioned technique with the creation and annihilation of cells both close to the boundaries of the region as well as in the neighbourhood of the shock wave front are presented in Fig.8 for the first case: at the initial instant the net consisted of 31 mesh points and, in the region encompassed by the motion, there were 1.1 mesh points ( + is for $t=4$, and 0 is for $t=8$ ). In the second case the total number of mesh points at the initial instant was 61 and of these 41 lay in the domain $x \leqslant x_{0}$. The error in determining the density did not exceed $5 \%$ in both cases. For comparison, we note that nets with a total number of mesh points $N=37-150$ were employed in analogous calculations in $/ 16 /$.

6. The problem of the decay of a strong discontinuity.

Among the solutions of the system of gas-dynamic equations, some are encountered which either contain a shock wave (index s.w.) or a contact discontinuity (index c.d.) In the problem of the breakdown of a strong discontinuity, there are simultaneously two types of discontinuities which move with constant velocities. This problem has been widely employed in the analysis of the quality of difference schemes by different authors $/ 16,39,40 \%$.

Let us formulate the problem in the following form: when $t=0$, let us put $p_{1}=480, \rho_{1}=8$, $u_{1}=0, p_{x}=1, \rho_{x}=1, u_{x}=0$, where values to the right and to the left of the discontinuity at the point $x=0$ are denoted by " $r$ " and " 1 ". The problem being considered is a selfmodelling problem wi.th respect to the variable $\xi=x / t$. The selfmodelling coordinates of the discontinuities have the following form: $\xi_{1}=-10, \quad \xi_{r}=1.0933, \quad \xi_{c} . d .=8.32, \quad \xi_{\text {s.w. }}=11.24(\gamma=9 / 3)$.

The exact solution in regions I-IV is described as foliows:

$\begin{array}{crcccc}\text { domain } & \text { I } & \text { II } & \text { III } & \text { IV } & \text { V } \\ \rho & 8 & (2-u / 15)^{3} & 3,0193 & 3.85 & 1 \\ u & 0 & 0.75(\xi+10) & 8.32 & 8.32 & 0 \\ p & 480 & 15 \rho^{1 / s} & 94.52 & 94.52 & 1\end{array}$

In the numerical calculations, 5 cells were chosen in each of the regions $I-V$. In region II, the mesh points of the net were moved along the $C$--characteristics, that is, the current $Q$ was selected in the form $Q=\rho u_{0}$. In the regions of piecewise-constant solutions, a a diffusion mechanism for pushing the mesh points apart: $Q=-D_{0} \partial \psi / \partial q$ was chosen. Profiles of the density at the instant of time $t=0.46$ are shown in Fig.9. The solid line is the exact solution and the small circles are the numerical solution. The number and position of the markers corresponds to the number and position of the mesh points of the net. The maximum error was observed in the region of the rarefaction wave and this was 0.58 .

Conclusion.

An analysis of the solutions of a number of well-known test problems (Sects.4-6) enables us to make the following assertions.

A finite-difference method has been proposed for solving non-slationary, spatially onedimensional problems of gas dynamics. The method is based on the use of adaptive nets which are obtained by a coordinate transformation which is determined by the required solution.

An efficient computational algorithm has been constructed on the basis of the proposed method which enables one to increase the accuracy of the calculations considerably (the error with respect to the exact solution was 1-58 for the different problems) with a simultaneous decrease in the total number of mesh points of the net by a factor of two to five compared with other methods.

The method enables one to determine the site and the moment of formation of discontinuous 
solutions with great accuracy.

Using this method, the fronts of strong, weak and contact discontinuities can be isolated almost precisely.

The method is economic and readily allows one to introduce a mechanism for generating mesh points.

The method reduces the requirements regarding the quality of the difference schemes. The adaptive nets used in this method, which are dynamically linked to the solution, lead to a reduction in the approximation viscosity and to the suppression of parasitic oscillations. The efficiency of the proposed method can be increased by using different optimization procedures which have not been carried out in the present paper.

\section{REFERENCES}

1. RICHTMYER R. and MORTON K., Difference Methods for Initial value problems, Mir, Moscow, 1972 .

2. SAMARSKII A.A. and POPOV YU.F., Difference Methods for the Solving Problems in Gas Dynamics, Nauka, Mosoow, 1980.

3. SCHULTZ V.D., Two-dimensional finite-difference hydrodynamic equations in Lagrange variables, in: Computational Methods in Hydrodynamics, Mir, Moscow, 9-54, 1972.

4. BATLER R., Development of the LING method, in: Numerical methods in Fluid Mechanics, Mir, Moscow, 146-155, 1973.

5. CROWLEY W., FLAG - a free Lagrangian method for the numerical modelling of hydrodynamic flows in two dimensions, in: Numerical Methods in Fluid Mcchanics, Mir, Moscow, 135-145, 1973.

6. BELOTSERKOVSKII O.M., Numerical Modelling in the Mechanics of Continuous Media, Nauka, Moscow, 1984

7. PASKONOV V.M., Difference schemes on a selforganizing sef of computational points in twodimensional simply connected regions of arbitrary form, zh. vychisl. Mat. mat. Fiz., Il, 3, 776-782, 1971 .

8. THOMFSON J.F., THAMES F.S. and MASTIN C.W., Automatic numerical generation of body-fitted curvilinear coordinate system for field containing any number of arbitrary two-dimensional bodies, J. Comput. Phys., 15, 229-319, 1974.

9. STEGER J.L., Implicit finite-difference simulation of flow about arbitrary two-dimensional geometrics, AIAA Journal, 16, 7, 679-686, 1978.

10. HINDMAN R.G., KUTLER P. and ANDERSON D., TWO-dimensional unsteady Euler equation solver for arbitrary shaped flow region, A.IAA Journal, 19, 4, 424-431, 1981.

11. GODUNOV S.K., ZABRODIN A.V., IVANOV M.YA. et al., Numerical Solution of Multidimensional Problems in Gas Dynamics, Nauka, Moscow, 1976.

12. BELOTSERKOVSKII O.M., GRUDNITSKII V.G. and RYGALIN V.N., Isolation of discontinuities in the calculation of one-dimensional non-stationary flows of a gas, Dokl. Akad. Nauk SSSR, $272,1,49-52,1983$.

13. HIRT G.W., AMSDEN A.A. and COOL J.L., An arbitrary Lagrangian-Eulerian computing method for all flow speeds, J. Comput. Phys., 14, 227-253, 1974.

14. VOLKOVA R.A., KRUGLYAKOVA L.V., MYSHETSKAYA E.E. et al., The ATLANT program for solving two-dimensional problems of controlled laser synthesis, Inst. Frikl. Matem. Akad. Nauk SSSR, 1985.

15. GOLOVIZNIN V.M., SAMARSKII A.A. and FAVORSKII A.P., A variational principle for obtaining the equations of magnetohydrodynamics in mixed Eulerian-Lagrangian variables, Zh. vychsil. Mat. mat. Fiz., 21, 2, 409-42.2, 1981 .

16. GOLOVIZNIN V.M., RYAZANOV M.A., SAMARSKII A.A. et al., Difference schemes of gas dynamics with balanced convective flows, in: Computational Methods in Mathematical physics, Izd. Moskovsk. Gos. Univ (MGU), Moscow, 5-41, 1986.

17. KOLDOVA A.V., KUZNETSOV O.A., POVESHCHENKO YU.A. and POPOV YU.P., An approach to solving problems in gas dynamics with a variable mass of a quasiparticle, Preprint 55, Inst. Prikl. Matem. Akad. Nauk SSSR, Moscow, 1985.

18. POVESHCHENKO YU.A. and POPOV YU.P., SOme problems of gas dynamics when there are sources, zh. vychisl. Mat. mat. Fiz., 18, 4, 1048-1056, 1978.

19. KOLDOVA A.V., KUZNETSOV O.A., POVESHCHENKO YU.A. et al., Completely conservative difference schemes for the equations of the mechanics of a continuous mcdium in quasi-Lagrangian variables in the presence of gravitational and magnetohydrodynamic processes, Freprint 55, Inst. Prik1. Matem. Akad. Nauk SSSR, Moscow, 1985.

20. VOLOSEVICH P.P., DAR'IN N.A., KARPOV V.YA. and KRUKOVSKII A.YU, , on the calculation of problems of magnetohydrodynamics with mass sinks in quasi-Lagrangian coordinates, Preprint 7, Inst. Prikl. Matem. Akad. Nauk SSSR, Moscow, 1984.

21. THONPSON J.F., Numerical Grid Generation, North-Holland, New York, 1982.

22. THOMPSON J.F., Grid generation techniques in computational fluid dynamics, AIAA Journal, 22 , I1, 1505-1523, 1984.

23. THOMPSON J.F., WARSI Z.U.A. and MASTIN C.W., Boundary fitted coordinate system for the numerical solution of partial differential equations - A review, J. Comput. Phys., 47, $1-108,1982$.

24. GELINAS R.J., DOSS S.K. and MILLER K., The moving finite element method: applications to general partial differential equations with multiple large gradients, J. Comput. Phys.. $40,202-249,1981$. 
25. COOK A.E. and LUNCAN R., A node-moving algorithm with application to Burger's equation and the Moltz problem, Appl. Math. Modeling, 6, 463-466, 1982.

26. KOVENYA V.M. and YANENKO N.N., A difference scheme on moving netw for solving the equations of a viscous gas, zh. vychisl. Mat. mat. Fiz., 19, 1, 174-188, 1979.

27. BELI J.B. and SHUBIN G.R., An adaptive grid finite difference method for conservation laws, J. Comput. Phys., 52, 569-591, 1983.

28. DEGMYAREV L.M. and DFOZDOV V.V., Nets in elliptic problems on a surface which adapt to the solution, Differents. Uravneniya, 20, 7, 1194-1203, 1984

29. RAI M.M. and ANDERSON D., Application of adaptive grid to fluid-flow problems with asymptotic solutions, AIAA Journal, 20, 4, 469-502, 1982.

30. DEGTYAREV L.M., DROZDOV V.V. and IVANOVA T.C., Method of adaptive grids in one-dimensional boundary value problems with a boundary layer, Preprint 164, Inst. Prikl. Matem. Akad. Nauk SSSR, Moscow, 1986.

31. DWYER H.A., KEE R.T. and SANDERS B.R., Adaptive grid method for problems in fluid mechanics and neat transfer, AIAA Journal, 18, 10, 1205-1212, 1980.

32. WINKLER K.-H.A., NORMAN M.I. and NEWMAN M.J., Adaptive mesh techniques for fronts in star formation, Max Planck Inst. Astron. und Astrophys. MPA-93, Uktober, 1983.

33. DAR'IN N.A. and MAZHUKIN V.I., Method of constructing adaptive grids for one-dimensional boundary value problems, Preprint 33, Inst. Prikl. Matem. Akad. Nauk SSSR, Moscow, 1987.

34. DAF'IN N.A. and MAZHURIN V.I., Mathematical modelling of the stcfan problem on an adaptive grid, Differents. Uravneniya, 23, 7, 1154-1159, 1987.

35. OVSYANNIKOV L.V., Lectures on the Principles of Gas Dynamics, Nauka, Moscow, 1981.

36. SHOKIN YU.I., The Method of Differential. Approximation, Nauka, Novosibirsk, 1979.

37. ROZHDESTVENSKII B.L. and YANENKO N.N., Systems of Quasilinear Equations, Nauka, Moscow, 1968.

38. ZEL'DOVICH YA.B. and RAIZER YU.P., The Physics of Shock waves and High Temperature Hydrodynamic Phenomena, Nauka, Moscow, 1966.

39. EORIS J.P., BOOK D.L. and HAIN K., Flux-corrected transport. II a: Generalization of the method, J. Comput. Phys., 18, 248-233, 1975.

40. ZHMAKIN A.I. and FURSENKO A.A., On a monotonic difference scheme of direct calculation, Zh. vychisl. Mat. mat. Fiz., 20, 4, 1021-1031, 1980. 